\title{
Parenchymal transection in robotic liver resection: results of 70 resections using the Vessel Sealer
}

\author{
Carolijn L. Nota, I. Quintus Molenaar, Wouter W. te Riele, Hjalmar C. van Santvoort, Inne H. M. Borel \\ Rinkes, Jeroen Hagendoorn
}

Department of Surgery, UMC Utrecht Cancer Center and Regional Academic Cancer Center Utrecht (RAKU), Utrecht 3584 CX, the Netherlands.

Correspondence to: Dr. Jeroen Hagendoorn, University Medical Center Utrecht, Heidelberglaan 100, Utrecht 3584 CX, the Netherlands. E-mail: j.hagendoorn-3@umcutrecht.nl

How to cite this article: Nota CL, Molenaar IQ, te Riele WW, van Santvoort HC, Borel Rinkes IHM, Hagendoorn J. Parenchymal transection in robotic liver resection: results of 70 resections using the Vessel Sealer. Mini-invasive Surg 2020;4:74.

http://dx.doi.org/: 10.20517/2574-1225.2020.57

Received: 14 Jun 2020 First Decision: 18 Jul 2020 Revised: 4 Aug 2020 Accepted: 11 Aug 2020 Published: 6 Nov 2020

Academic Editor: Giulio Belli Copy Editor: Cai-Hong Wang Production Editor: Jing Yu

\begin{abstract}
Aim: There is no standard technique for transection of the hepatic parenchyma during robotic liver resection. The aim of this study was to describe the outcomes of robotic liver resections using the Vessel Sealer for parenchymal transection.
\end{abstract}

Methods: This is a post hoc analysis of a prospective database. All consecutive patients who underwent robotic liver resection in the Regional Academic Cancer Centre, Utrecht, Netherlands, between August 2015 and January 2019 were included.

Results: A total of 70 robotic liver resections were performed, including 60 minor resections (86\%) and ten hemihepatectomies (14\%). Five procedures (7\%) were converted. Mean parenchymal transection time was 43 \pm 26 min. Median blood loss was $150 \mathrm{~mL}$ (interquartile range 40-300). Ten patients (14\%) suffered from a major complication, and three patients (4\%) had bile leakage postoperatively. One patient died from post-hepatectomy liver failure.

Conclusion: Based on the results of this series, consisting of 60 minor liver resections and 10 hemihepatectomies, we conclude that the use of the Vessel Sealer during the parenchymal transection in liver resection is feasible and safe.

\footnotetext{
Cc) (1) (C) The Author(s) 2020. Open Access This article is licensed under a Creative Commons Attribution 4.0 International License (https://creativecommons.org/licenses/by/4.0/), which permits unrestricted use, sharing, adaptation, distribution and reproduction in any medium or format, for any purpose, even commercially, as long as you give appropriate credit to the original author(s) and the source, provide a link to the Creative Commons license, and indicate if changes were made.
}

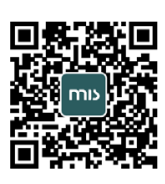


Keywords: Robotic liver resection, minimally-invasive hepatectomy, robot-assisted surgery, robotic surgery, hepatic resection

\section{INTRODUCTION}

The benefits to the patient of a minimally invasive approach to liver resection include fewer complications, less blood loss, and an enhanced recovery after surgery ${ }^{[1]}$. Conventional laparoscopy, however, has technical limitations. Laparoscopic instruments have a straight work-axis and, therefore, have limited freedom of movement. To overcome these impairments the surgical robot was introduced, which provides articulating instruments, a 3-dimensional view, and scaled movements ${ }^{[2,3]}$. Several studies have shown the safety and feasibility of robotic liver resection ${ }^{[4]}$.

During liver resection, transection of the hepatic parenchyma forms an essential part of the procedure. Inadequate sealing of vascular and biliary structures can result in bile leakage or bleeding, potentially causing postoperative complications and mortality. Several techniques and devices have been developed for parenchymal transection, such as the clamp crushing technique, cavitron ultrasonic surgical aspirator (CUSA) (Integra LifeSciences, Tullamore, Ireland), ultrasonic devices, staplers and mono- and bipolar devices $^{[5,6]}$. Most of these techniques were developed for, and are predominantly used in, open surgery. In laparoscopic liver surgery, the transection is mostly performed using CUSA, sealing devices and staplers. For robotic surgery, it has not yet been determined which device is best suited for parenchymal transection. Currently, the robotic Harmonic Scalpel (Intuitive Surgical, Sunnyvale, California, USA) or robotic bipolar cautery (Maryland Bipolar Forceps, Intuitive Surgical, Sunnyvale, California, USA) are the most frequently reported devices used for parenchymal transection during robotic liver resection ${ }^{[7]}$. However, the robotic Harmonic Scalpel lacks the ability to articulate and the Maryland Bipolar Forceps seems not optimally suited for larger transection planes.

The EndoWrist ${ }^{\oplus}$ One ${ }^{\mathrm{TM}}$ Vessel Sealer (on the Xi/X robotic systems: EndoWrist ${ }^{\oplus} \mathrm{One}^{\mathrm{TM}}$ Vessel Sealer Extend) (Intuitive Surgical Inc., Sunnyvale, CA, USA) is a fully wristed robotic energy device $\left(60^{\circ}\right.$ of articulation in all directions for the Extend) that is approved to seal and cut vessels up to $7 \mathrm{~mm}$ in diameter. The aim of this study is to report the technical details and clinical outcomes of a series of consecutive robotic liver resections during which the Vessel Sealer was used for parenchymal transection.

\section{METHODS}

\section{Study design}

This is a post hoc analysis of a prospective database. In addition, recordings of the surgical procedures were reviewed retrospectively for determination of parenchymal transection duration. All consecutive patients who underwent robotic liver resection in the Regional Academic Cancer Centre Utrecht (RAKU) at both University Medical Centre Utrecht and St. Antonius Hospital Nieuwegein, between 1st August 2015 and 11th January 2019, were included. Patients were selected for robotic liver resection in a multidisciplinary board meeting. As this case series also reflects a learning curve of robotic hepatectomy starting with easy minor resections and progressing to difficultly-located minor resections, and eventually hemihepatectomy, no uniform inclusion criteria are applicable. In general, exclusion criteria for the robotic approach in this series were extended liver resection ( $>4$ segments), tumour adjacent to the inferior vena cava or hepatic vein insertions, and perihilar cholangiocarcinoma. In the first cases, cirrhosis was a relative contraindication (unless minor/wedge resection) but, with growing experience, this was no longer considered a contraindication. 
We adhered to the Strengthening the Reporting of Observational Studies in Epidemiology (STROBE) Statement ${ }^{[8]}$. Eleven of the minor resections of the posterosuperior liver segments have been described previously within a multi-institutional cohort study ${ }^{[9]}$. The overall initial experience at our centre has been published previously, including surgical outcomes of the first eighteen procedures ${ }^{[3,10]}$.

\section{Definitions}

Liver segments were defined using Couinaud's classification ${ }^{[11]}$. Segments 2, 3, 4B, 5 and 6 were classified as anterolateral segments; segments 1, 4A, 7 and 8 were classified as posterosuperior segments. Minor liver resection was defined as the resection of three or less segments, while major liver resection was defined as the resection of four or more segments. A wedge resection was counted as a half segment ${ }^{[12]}$. En-bloc resections of the adrenal gland or diaphragm and cholecystectomies were not considered concomitant procedures. Operative time was defined as time from first incision until wound closure. Postoperative complications were scored using the Clavien-Dindo (CD) grading system for postoperative complications ${ }^{[13]}$. Major complications were defined as CD grade III or higher. Bile leak was defined using the International Study Group of Liver Surgery definition and grading system ${ }^{[14]}$. Complications were scored during index admission. If a patient was readmitted within ten days after discharge, this readmission was still considered index admission. Conversion was defined as a laparotomy made for any reason other than for specimen extraction. Resections were considered radical (Ro) if no tumour cells were present in the transection surface and within $1 \mathrm{~mm}$ of the transection surface. Resections were considered irradical (R1) if tumour cells were present in the transection surface or within $1 \mathrm{~mm}$ of the transection surface ${ }^{[15]}$. If multiple tumours were resected, the closest margin determined the R status.

\section{Data collection}

The baseline patient characteristics collected were the year of surgery, age, sex, body mass index, American Society of Anesthesiologists score, previous abdominal surgery, and indication for resection. Data on details of the operation collected included the resection performed, concomitant procedures, operative time, console time, parenchymal transection time, estimated blood loss, conversion, placement of surgical drain, use of Pringle manoeuvre, duration of inflow occlusion, epidural analgesia, number of stapler loads used per procedure, type of robotic system, definitive histopathological diagnosis, margin status, and tumour size. Postoperative outcomes were CD grade III or higher complications, bile leakage, unplanned ICU admission, relaparotomy, percutaneous or endoscopic catheter drainage, length of hospital stay, readmission, 30-day mortality, 90-day mortality and trocar herniation during 1-year follow-up.

\section{Comparison with conventional laparoscopic approach}

Additionally, to put our results into perspective and to compare outcomes of our series of robotic liver resections to conventional laparoscopy, we have provided an overview of the outcomes of all laparoscopic liver resections performed in the Netherlands between 2011 and 2016. Data were extracted from the Dutch nationwide LAELIVE database on minimally invasive liver surgery ${ }^{[16]}$ (published in part).

\section{Statistical analysis}

Data with a normal distribution were reported as mean with standard deviation (SD). Data with a skewed distribution were reported as median with interquartile range (IQR). Missing values were reported for each parameter.

\section{Ethical approval}

The Medical Ethics Review Committee approved the study protocol with a waiver for informed consent.

\section{Parenchymal transection technique}

In the majority of procedures, parenchymal transection began with ultrasound for delineation of the oncological margin. Either a laparoscopic ultrasound probe was used or a robotic 'drop-in' probe (both: 
Table 1. Patient characteristics

\begin{tabular}{ll}
\hline Parameter & Outcome \\
\hline Year of surgery, $n(\%)$ & $3(4)$ \\
2014 & $9(13)$ \\
2015 & $9(13)$ \\
2016 & $19(27)$ \\
2017 & $28(40)$ \\
2018 & $2(3)$ \\
2019 (up to January 11th) & $60(14)$ \\
Age, mean (SD), years & $35(50)$ \\
Sex, male, $n(\%)$ & $27(5)$ \\
BMI, mean (SD), kg/m ${ }^{2}$ & \\
ASA score, $n(\%)^{1}$ & $3(4)$ \\
ASA 1 & $49(70)$ \\
ASA 2 & $16(23)$ \\
ASA 3 & $45(64)$ \\
Previous abdominal surgery, $n(\%)$ & $6(9)$ \\
Redo liver resection, $n(\%)$ & \\
Indication for resection, $n(\%)$ & $32(46)$ \\
CRLM & $7(10)$ \\
Metastases, other & $16(23)$ \\
HCC & $5(7)$ \\
Cholangiocarcinoma & $10(14)$ \\
Other &
\end{tabular}

${ }^{1}$ Two missing values. SD: standard deviation; BMI: body mass index; ASA: American Society of Anesthesiologists; CRLM: colorectal liver metastases; HCC: hepatocellular carcinoma

Hitachi Aloka Medical Inc., Wallingford, CT, USA). The latter provides more freedom of movement and hence facilitates imaging of the posterosuperior segments more easily. A Pringle manoeuvre was applied when deemed appropriate. The Vessel Sealer (Extend) was combined with the Maryland Bipolar Forceps and Fenestrated Bipolar Forceps. The Vessel Sealer was employed by clamp-crushing thin layers of tissue (as much as possible under direct vision to avoid lacerations of small veins and bile ducts) with subsequent double sealing and cutting, working in layers from superficial to deep in the liver parenchyma as shown previously ${ }^{[17,18]}$. Hem-o-lok clips (Teleflex Inc., Morrisville, NC, USA) or laparoscopic Endo GIA (Medtronic, Minneapolis, MN, USA) were used for control of the hepatic pedicles and larger branches of the hepatic veins, where appropriate.

\section{RESULTS}

In total, 70 resections were performed in 68 patients. Two patients underwent robotic liver resection twice for recurrent hepatocellular carcinoma.

\section{Patient characteristics}

Patient characteristics are summarized in Table 1. The majority of liver resections was performed for colorectal liver metastases $(n=32 ; 46 \%)$.

\section{Operative characteristics and histopathological outcomes}

Details on the surgical procedures and pathology are provided in Table 2. Five procedures were converted to laparotomy, for several reasons: in three cases there was a lack of anatomical overview during transection of the hepatic parenchyma; one patient had severe intra-abdominal adhesions; and in one patient a safe oncological margin could not be assured robotically.

In all procedures the Vessel Sealer was used for parenchymal transection. In 22 procedures (31\%) stapling devices were also used to control the hepatic pedicles; these resections were left lateral sectionectomies 
Table 2. Operative characteristics and histopathological outcomes

\begin{tabular}{|c|c|}
\hline Parameter & Outcome \\
\hline \multicolumn{2}{|l|}{ Resections performed, $n(\%)$} \\
\hline Minor resection solely including anterolateral segments & $32(46)$ \\
\hline Wedge resection & $17(24)$ \\
\hline Segmental resection & $15(21)$ \\
\hline Minor resection including posterosuperior segments & $28(40)$ \\
\hline Wedge resection & $21(30)$ \\
\hline Segmental resection & $7(10)$ \\
\hline Major resection (right and left hepatectomy) & $10(14)$ \\
\hline \multicolumn{2}{|l|}{ Surgical details } \\
\hline Concomitant procedures, $n(\%)$ & $7(10)$ \\
\hline Operative time, mean (SD), $\min ^{1, \wedge}$ & $160(78)$ \\
\hline Console time, mean (SD), $\min ^{2, \star}$ & $111(69)$ \\
\hline Parenchymal transection time, mean (SD), $\min ^{3, \star}$ & $43(26)$ \\
\hline $\mathrm{EBL}$, median (IQR), $\mathrm{mL}$ & $150(40-300)$ \\
\hline RBC transfusions, median (IQR) & $0(0-0)$ \\
\hline FFP transfusions, median (IQR) & $0(0-0)$ \\
\hline Conversion to laparotomy, $n$ (\%) & $5(7)$ \\
\hline Placement of surgical drain, $n(\%)$ & $27(38)$ \\
\hline Use of biological agents (TachoSil, Surgicel), $n(\%)^{\star}$ & $51(79)$ \\
\hline Pringle manoeuvre performed (intermittent clamping), $n$ (\%) & $31(44)$ \\
\hline Duration of inflow occlusion, mean (SD) $\min ^{1}$ & $41(15)$ \\
\hline Epidural analgesia, $n(\%)$ & $20(29)$ \\
\hline Stapler loads used per procedure, median (IQR)* & $0(0-2)$ \\
\hline \multicolumn{2}{|l|}{ Robotic system used, $n(\%)$} \\
\hline da Vinci Si surgical system & $55(79)$ \\
\hline da Vinci $X$ surgical system & $6(9)$ \\
\hline da Vinci Xi surgical system & $9(13)$ \\
\hline \multicolumn{2}{|l|}{ Histopathological outcomes, $n(\%)$} \\
\hline \multicolumn{2}{|l|}{ Definitive diagnosis } \\
\hline CRLM & $31(44)$ \\
\hline Metastases, other & $5(7)$ \\
\hline $\mathrm{HCC}$ & $15(21)$ \\
\hline Intrahepatic Cholangiocarcinoma & $4(6)$ \\
\hline Benign & $13(19)$ \\
\hline Other & $2(3)$ \\
\hline Cirrhosis on final pathology, $n(\%)$ & $8(11)$ \\
\hline Radical (RO) resection ${ }^{\#}$ & $42(76)$ \\
\hline Tumor size, mean (SD), $\mathrm{mm}^{\&}$ & $37(26)$ \\
\hline
\end{tabular}

'One missing value; ${ }^{2}$ four missing values; ${ }^{3}$ twenty missing values; ’ operative time for liver resection, corrected for concomitant procedures; ${ }^{*}$ converted cases excluded; ${ }^{*}$ solely reported for malignancies; ${ }^{\varepsilon_{i}}$ cases of multiple resected tumours, only the largest tumour was included in the calculation. SD: standard deviation; IQR: interquartile range; RBC: red blood cells; FFP: fresh frozen plasma; CRLM: colorectal liver metastases; HCC: hepatocellular carcinoma; EBL: estimated blood loss

$(n=8)$, left or right hepatectomies $(n=8)$, resections of the posterior sector $(n=3)$, and resections of segment 7 or $8(n=3)$. Overall, median blood loss was $150 \mathrm{~mL}$ (IQR 40-300), and in 51 procedures (79\%) biological agents were applied to the resection surface to ensure haemostasis and biliostasis when deemed appropriate. No technical errors or handling difficulties of the Vessel Sealer were encountered.

\section{Postoperative outcomes}

Postoperative outcomes are summarized in Table 3. Ten patients (14\%) suffered from a major complication. Three patients (4\%) suffered from bile leakage postoperatively, two of which underwent a left hepatectomy, and the third patient underwent a segmental resection of segment 5 . Of the three patients who suffered from bile leakage, two patients needed additional radiological drainage. The median length of hospital stay was four days. In total, 37 patients (53\%) were discharged on day 4 or earlier; 12 patients (17\%) went home on postoperative day one or two. 
Table 3. Postoperative outcomes

\begin{tabular}{ll}
\hline Parameter & Outcome \\
\hline Major complication, $n$ (\%) & $10(14)$ \\
Clavien-Dindo grade III a/b & $7(10)$ \\
Non-bilious fluid collection, drained radiologically & $2(3)$ \\
Non-bilious fluid collection, drained laparoscopically & $1(1)$ \\
Herniated omentum, closed under local anesthesia & $1(1)$ \\
Bilious fluid collection, drained radiologically & $2(3)$ \\
Trocar herniation, corrected surgically & $1(1)$ \\
Clavien-Dindo grade IV a/b & $2(3)$ \\
ICU admission for respiratory insufficiency & $2(3)$ \\
Bile leakage, $n$ (\%) & $3(4)$ \\
Grade A & $1(1)$ \\
Grade B & $2(3)$ \\
ICU admission, $n$ (\%) & $5(7)$ \\
Unplanned ICU admission, $n(\%)$ & $3(4)$ \\
Relaparotomies, $n(\%)$ & $0(0)$ \\
Minimally invasive drainages, $n(\%)$ & $5(7)$ \\
Length of stay, median (IQR), days & $4(3-6)$ \\
Readmission within 10 days, $n(\%)$ & $4(6)$ \\
Readmission within 90 days, $n(\%)$ & $6(9)$ \\
$30-$ day mortality, $n(\%)$ & $1(1)$ \\
$90-$ day mortality, $n(\%)$ & $1(1)$ \\
Trocar herniation within one year after surgery requiring surgical intervention, $n(\%)$ & $2(3)$ \\
\hline
\end{tabular}

ICU: intensive care unit; IQR: interquartile range

One patient died postoperatively due to post hepatectomy liver failure. The patient had a past medical history of hepatitis B, no signs of cirrhosis or portal hypertension in preoperative hepatology evaluation, and underwent right hepatectomy for a hepatocellular carcinoma. Due to the lack of anatomical overview during parenchymal transection, the procedure was converted to open hemihepatectomy. Postoperatively, the patient suffered from grade $\mathrm{C}$ posthepatectomy liver failure progressing to multiple organ failure and death on postoperative day 12. Definitive pathology showed a hepatocellular carcinoma as well as liver cirrhosis.

\section{Comparison to conventional laparoscopy}

A summary of several outcomes from our series and an overview of the outcomes of all laparoscopic liver resections performed in the Netherlands between 2011 and 2016 are provided in Table 4. In total, 885 conventional laparoscopic liver resections were performed, of which 683 (77\%) were minor resections. Mean operative time was $164 \mathrm{~min}$ (SD 95) for the conventional laparoscopic liver resections and median blood loss was $200 \mathrm{~mL}$ (IQR 50-500). A total of 121 procedures (14\%) were converted to laparotomy and 76 patients (9\%) suffered from a major complication. Nine patients (1\%) died after conventional laparoscopic liver resection. Outcomes of our robotic liver resections are comparable to the outcomes of all conventional laparoscopic liver resections performed between 2011 and 2016 in the Netherlands.

\section{DISCUSSION}

In this study we report the surgical details and clinical outcomes of 70 consecutive robotic liver resections in which the Vessel Sealer was used for parenchymal transection. Our results demonstrate that the use of this device facilitates safe transection of the hepatic parenchyma, without compromising postoperative clinical outcomes. No postoperative bleeding occurred and only three patients (4\%) suffered from bile leakage postoperatively.

Over the past decade, robotic surgery has become an important alternative to conventional laparoscopy. Recently, a nationwide trend in the US towards an increase of the use of robotic surgery has been observed 
Table 4. Summarized comparison of robotic liver resection with conventional laparoscopic liver resection

\begin{tabular}{lccccccc}
\hline Approach & $\boldsymbol{n}$ & $\begin{array}{c}\text { Minor resections, } \\
\boldsymbol{n}(\%)\end{array}$ & $\begin{array}{c}\text { Operative time, } \\
\text { mean (SD), } \mathbf{m i n}\end{array}$ & $\begin{array}{c}\text { Blood loss, median } \\
(\text { IQR), mL }\end{array}$ & $\begin{array}{c}\text { Conversion, } \\
\boldsymbol{n}(\%)\end{array}$ & $\begin{array}{c}\text { Major complications, } \\
\boldsymbol{n}(\%)\end{array}$ & $\begin{array}{c}\text { Mortality, } \\
\boldsymbol{n}(\%)\end{array}$ \\
\hline $\begin{array}{l}\text { Conventional } \\
\text { laparoscopy }\end{array}$ & 885 & $683(77)^{\star}$ & $164(95)$ & $200(50-500)$ & $121(14)$ & $76(9)$ & $9(1)$ \\
$\begin{array}{l}\text { Robotic liver } \\
\text { resection }\end{array}$ & 70 & $60(86)$ & $160(78)$ & $150(40-300)$ & $5(7)$ & $10(14)$ & $1(1)$ \\
\hline
\end{tabular}

*Defined as less than three liver segments in Dutch LAELIVE database; ^defined using the Accordion severity grading system of surgical complications. SD: standard deviation; IQR: interquartile range

for pancreatoduodenectomy, whilst the number of conventional laparoscopic pancreatoduodenectomies performed decreased ${ }^{[19]}$. This finding supports the hypothesis that robotic surgery might be better suited (and more widely implemented) than conventional laparoscopy for complex procedures, such as pancreatic resection or liver resection.

Since the use of robotic technology in liver resection is gaining momentum, new techniques and devices for parenchymal transection have emerged. Initial series on robotic liver resection mostly reported the use of the robotic Harmonic Scalpel or the Maryland Bipolar Forceps for transection of the parenchyma ${ }^{[7]}$. Other currently available devices include the PK Dissecting Forceps (Intuitive Surgical, Sunnyvale, California, USA), EndoClips, robotic stapler, and the Vessel Sealer ${ }^{[20]}$. The Harmonic Scalpel, however, lacks the ability to articulate. The Maryland Bipolar Forceps and the PK Dissecting Forceps provide meticulous dissection, but these instruments appear inefficient for larger transection planes. EndoClips provide reliable ligation of vessels and bile ducts, though do not seem efficient for larger transection planes. Robotic staplers facilitate reliable sealing, but are expensive. A few cases using the Vessel Sealer for transection of the parenchyma during robotic liver resection have been reported by Kingham et al. ${ }^{[21]}$, however, no separate outcomes were reported for the different transection techniques used in this study.

The results in our study demonstrate that the use of the Vessel Sealer is feasible and safe during robotic liver resection. Only ten patients (14\%) suffered from a major complication, from which one patient died. However, this patient suffered from post hepatectomy liver failure, which is most likely a consequence of the extent of the resection rather than of the parenchymal transection technique chosen. Three patients (4\%) suffered postoperatively from bile leakage, which is comparable to large series reporting on open and laparoscopic liver resection ${ }^{[22-25]}$. We could generally employ the Vessel Sealer for parenchymal bile ducts, portal branches and veins but use a stapler and/or hemolocks for inflow/outflow pedicles, major veins, or when larger vascular structures are encountered that are clearly beyond a size that could easily be sealed with a margin within the length of sealer's surface at 90 degrees. We therefore conclude that the Vessel Sealer is appropriate to seal most vascular structures encountered within the parenchyma of the liver segments. The R1 resection rate in our series (defined as a surgical margin of $<1 \mathrm{~mm}$ ) appears to be relatively high (24\%). However, studies show that R1 resection for colorectal liver metastases (CLRM) can be considered acceptable ${ }^{[26,27]}$. The majority of our R1 resections were for CLRM. In addition, in our initial series, robotic manipulation of the liver tissue during resection may have caused inadvertent laceration in the specimen contributing to the number of R1 margins on final pathology in several cases.

Secondly, we provided an overview of all conventional laparoscopic liver resections performed in the Netherlands. Our outcomes are not inferior to those of conventional laparoscopic liver resection. Major morbidity appeared to be lower after conventional laparoscopic liver resection, however, different definitions were used for the grading of the postoperative complications.

Several limitations must be considered for this study. Firstly, the patients who underwent robotic liver resection in this study were selected. Patients with tumours adjacent to the hepatic vessels, patients who 
underwent extended hepatectomies ( $>4$ segments), or patients who had a past medical history of extensive abdominal surgery, were in general not deemed fit for a robotic approach. Although our resections might not fully represent the entire spectrum of liver resections, there were ten major resections performed (14\%) and indications varied widely, including patients with cirrhosis (11\%). Moreover, 45 patients (64\%) were selected who underwent previous abdominal surgery, including previous liver surgery in 6 patients. Second, some surgeons consider the tip of the Vessel Sealer to be too bulky and prefer a more refined instrument for transection of the parenchyma and dissecting out hepatic structures. The updated version of the Vessel Sealer, the Vessel Sealer Extend, however, has a slimmer jaw profile and therefore allows for more delicate dissection. Third, the retrospective nature of the study holds an inherent risk of bias. The comparison we conducted with conventional laparoscopy is obviously weaker than a head-to-head comparison. However, since the outcomes of all laparoscopic liver resections performed in the Netherlands are provided, these results reflect the true outcomes after conventional laparoscopic liver resection.

Based on the results of this series, consisting of 60 minor liver resections and 10 hemihepatectomies, we conclude that the use of the Vessel Sealer during the parenchymal transection in liver resection is feasible and safe.

\section{DECLARATIONS}

\section{Acknowledgments}

The authors would like to thank Prof. Yuman Fong (Department of Surgery, City of Hope Medical Center, Los Angeles) for his advice and support during the set-up of the robotic liver surgery program at University Medical Center Utrecht. The authors also like to thank Prof. Dr Marc G. H. Besselink and Dr Burak Gorgec (both of Department of Surgery, Amsterdam UMC, location: AMC) for providing additional data from their study.

\section{Authors' contributions}

Made substantial contributions to the design of the work, the data acquisition and analysis, and drafting of the manuscript: Nota CL, Molenaar IQ, te Riele WW, van Santvoort HC, Borel Rinkes IHM, Hagendoorn J

\section{Availability of data and materials}

Data are extracted from a prospectively maintained, secured institutional database. Due to the institution's privacy regulation, raw data won't be shared online.

\section{Financial support and sponsorship}

None.

\section{Conflicts of interest}

Prof. Dr. I. Quintus Molenaar and Dr. Jeroen Hagendoorn are proctor for Intuitive Surgical (Intuitive Surgical Inc., Sunnyvale, CA, USA). The content of this study is solely the responsibility of the authors and does not necessarily represent the official views of Intuitive Surgical. All other authors have declared no conflict of interest.

\section{Ethical approval and consent to participate}

Data were extracted from an anonymized database. Hence the study was waived from informed consent.

\section{Consent for publication}

Not applicable. 


\section{Copyright}

(c) The Author(s) 2020.

\section{REFERENCES}

1. Fretland ÅA, Dagenborg VJ, Bjørnelv GMW, et al. Laparoscopic versus open resection for colorectal liver metastases: the OSLOCOMET randomized controlled trial. Ann Surg 2018;267:199-207.

2. Leung U, Fong Y. Robotic liver surgery. Hepatobiliary Surg Nutr 2014;3:288-94.

3. Nota CL, Borel Rinkes IHM, Hagendoorn J. Setting up a robotic hepatectomy program: a Western-European experience and perspective. Hepatobiliary Surg Nutr 2017;6:239-45.

4. Nota CL, Borel Rinkes IHM, Molenaar IQ, et al. Robot-assisted laparoscopic liver resection: a systematic review and pooled analysis of minor and major hepatectomies. HPB (Oxford) 2016;18:113-20.

5. Pamecha V, Gurusamy KS, Sharma D, Davidson BR. Techniques for liver parenchymal transection: a meta-analysis of randomized controlled trials. HPB (Oxford) 2009;11:275-81.

6. Otsuka Y, Kaneko H, Cleary SP, et al. What is the best technique in parenchymal transection in laparoscopic liver resection? Comprehensive review for the clinical question on the 2nd International Consensus Conference on Laparoscopic Liver Resection. $J$ Hepatobiliary Pancreat Sci 2015;22:363-70.

7. Ho CM, Wakabayashi G, Nitta H, et al. Systematic review of robotic liver resection. Surg Endosc 2012;27:732-9.

8. von Elm E, Altman DG, Egger M, et al. The Strengthening the Reporting of Observational Studies in Epidemiology (STROBE) Statement: guidelines for reporting observational studies. Int J Surg 2014;12:1495-9.

9. Nota CL, Woo Y, Raoof M, et al. Robotic versus open minor liver resections of the posterosuperior segments: a multinational, propensity score-matched study. Ann Surg Oncol 2019;26:583-90.

10. Nota CLMA, Molenaar IQ, van Hillegersberg R, Borel Rinkes IHM, Hagendoorn J. Robotic liver resection including the posterosuperior segments: initial experience. $J$ Surg Res 2016;206:133-8.

11. Couinaud C. Liver lobes and segments: notes on the anatomical architecture and surgery of the liver. Presse Med 1954;62:709-12. (in French)

12. Reddy SK, Barbas AS, Turley RS, et al. A standard definition of major hepatectomy: resection of four or more liver segments. HPB (Oxford) 2011;13:494-502.

13. Dindo D, Demartines N, Clavien PA. Classification of surgical complications: a new proposal with evaluation in a cohort of 6336 patients and results of a survey. Ann Surg 2004;240:205-13.

14. Koch M, Garden OJ, Padbury R, et al. Bile leakage after hepatobiliary and pancreatic surgery: a definition and grading of severity by the international study group of liver surgery. Surgery 2011;149:680-8.

15. The Royal College of Pathologists. Dataset for histopathology reporting of liver resection specimens (including gall bladder) and liver biopsies for primary and metastatic carcinoma. Available from: https://www.rcpath.org/uploads/assets/cbae24ce-220c-4d9f95c5b83dd1 f0ad2b/Dataset-for-histopathology-reporting-of-liver-resection-specimens-and-liver-biopsies-for-primary-and-metastaticcarcinoma-2nd-edition.pdf. [Last accessed on 13 Aug 2020]

16. van der Poel MJ, Fichtinger RS, Bemelmans M, et al. Implementation and outcome of minor and major minimally invasive liver surgery in the Netherlands. HPB (Oxford) 2019;21:1734-43.

17. Nota CLMA, Molenaar IQ, Borel Rinkes IHM, Hagendoorn J. Robotic right hepatectomy for a central liver tumor- a video of the surgical technique. Surg Oncol 2019;30:108.

18. Nota CLMA, Molenaar IQ, Borel Rinkes IHM, Hagendoorn J. Robotic liver resection of segment 7: a step-by-step description of the technique. Surg Oncol 2020;34:206-7.

19. Panni RZ, Guerra J, Hawkins WG, et al. National pancreatic fistula rates after minimally invasive pancreaticoduodenectomy are improving with time: a NSQIP analysis. J Am Coll Surg 2019;229:192-9.e1.

20. Thakkar R, Kanwar A, Alessandri G, et al. Techniques of hepatic transection in robotic surgery - is there still scope for improvement? Adv Res Gastroentero Hepatol 2018;10:555781.

21. Kingham TP, Leung U, Kuk D, et al. Robotic liver resection: a case-matched comparison. World J Surg 2016;40:1422-8.

22. Donadon M, Costa G, Cimino M, et al. Diagnosis and management of bile leaks after hepatectomy: results of a prospective analysis of 475 hepatectomies. World J Surg 2016;40:172-81.

23. Yamashita Y, Hamatsu T, Rikimaru T, et al. Bile leakage after hepatic resection. Ann Surg 2001;233:45-50.

24. Nguyen KT, Gamblin TC, Geller DA. World review of laparoscopic liver resection-2,804 patients. Ann Surg 2009;250:831-41.

25. Takahashi H, Akyuz M, Aksoy E, et al. A new technique for hepatic parenchymal transection using an articulating bipolar $5 \mathrm{~cm}$ radiofrequency device: results from the first 100 procedures. HPB (Oxford) 2018;20:829-33.

26. Sadot E, Groot Koerkamp B, Leal JN, et al. Resection margin and survival in 2368 patients undergoing hepatic resection for metastatic colorectal cancer: surgical technique or biologic surrogate? Ann Surg 2015;262:476-85; discussion 483-5.

27. Viganò L, Costa G, Cimino MM, et al. R1 resection for colorectal liver metastases: a survey questioning surgeons about its incidence, clinical impact, and management. J Gastrointest Surg 2018;22:1752-63. 